\title{
A Big Toe in the Ear? Two Cases of Gouty Tophi Located in the Temporomandibular Joint
}

JOANNE GUERLAIN, JG, MD, AP-HP, Hopital Pitie-Salpetriere, Service de Chirurgie Maxillo-faciale et de Stomatologie; PATRICK GOUDOT, PG, MD, AP-HP, Hopital Pitie-Salpetriere, Service de Chirurgie Maxillo-faciale et de Stomatologie, and Sorbonne Universités, UPMC Univ Paris 06; THOMAS SCHOUMAN, TS, MD, AP-HP, Hopital Pitie-Salpetriere, Service de Chirurgie Maxillo-faciale et de Stomatologie, Paris, France. Address correspondence to Dr. T. Schouman, Service de Chirurgie Maxillo-faciale et de Stomatologie, AP-HP, Hopital Pitie-Salpetriere, 47-83 Boulevard de l'Hôpital, 75651 Paris Cedex 13, France. E-mail: thomas.schouman@psl.aphp.fr. J Rheumatol 2015;42:1261-2; doi:10.3899/jrheum.141578

Gout can occur in an unusual location without hyperuricemia and with heterogeneous $\mathrm{T} 1$ and $\mathrm{T} 2$ hyposignal.

A 44-year-old male patient consulted for an isolated and firm swelling of the pretragal region. A 72-year-old female patient presented with left pretragal tenderness and limited mouth opening. Both patients presented with multiple temporomandibular joint (TMJ) calcifications on computed tomography (CT), and heterogeneous $\mathrm{T} 1$ and $\mathrm{T} 2$ hyposignal TMJ lesions on magnetic resonance imaging (MRI; Figure 1 and Figure 2). Fine needle aspiration was performed for the first patient, but was not conclusive. Both patients underwent tumor resection. The pathological analysis allowed identifying gouty tophi. Both patients had normal serum uric acid concentration.
To our knowledge, only 8 out of the 11 previously reported cases of TMJ gouty tophi were proven histologically ${ }^{1}$. None of the 6 fine needle aspirations performed in these cases (including ours) were conclusive, whereas the sensitivity and specificity of tophus aspirates commonly range from $63 \%$ to $78 \%$ and from $93 \%$ to $100 \%$, respectively ${ }^{2}$. Six patients (including ours) were tested for uric acid concentration, which was always in the normal range. Hyperuricemia is the most important risk factor for gout, but some individuals may present with gout without hyperuricemia ${ }^{2}$. Imaging is not specific. A CT scan usually shows intra- or extraarticular calcified bodies that most often suggest chondromatosis in this location. The MRI presentation of tophi is nonspecific (intermediate $\mathrm{T} 1$ and

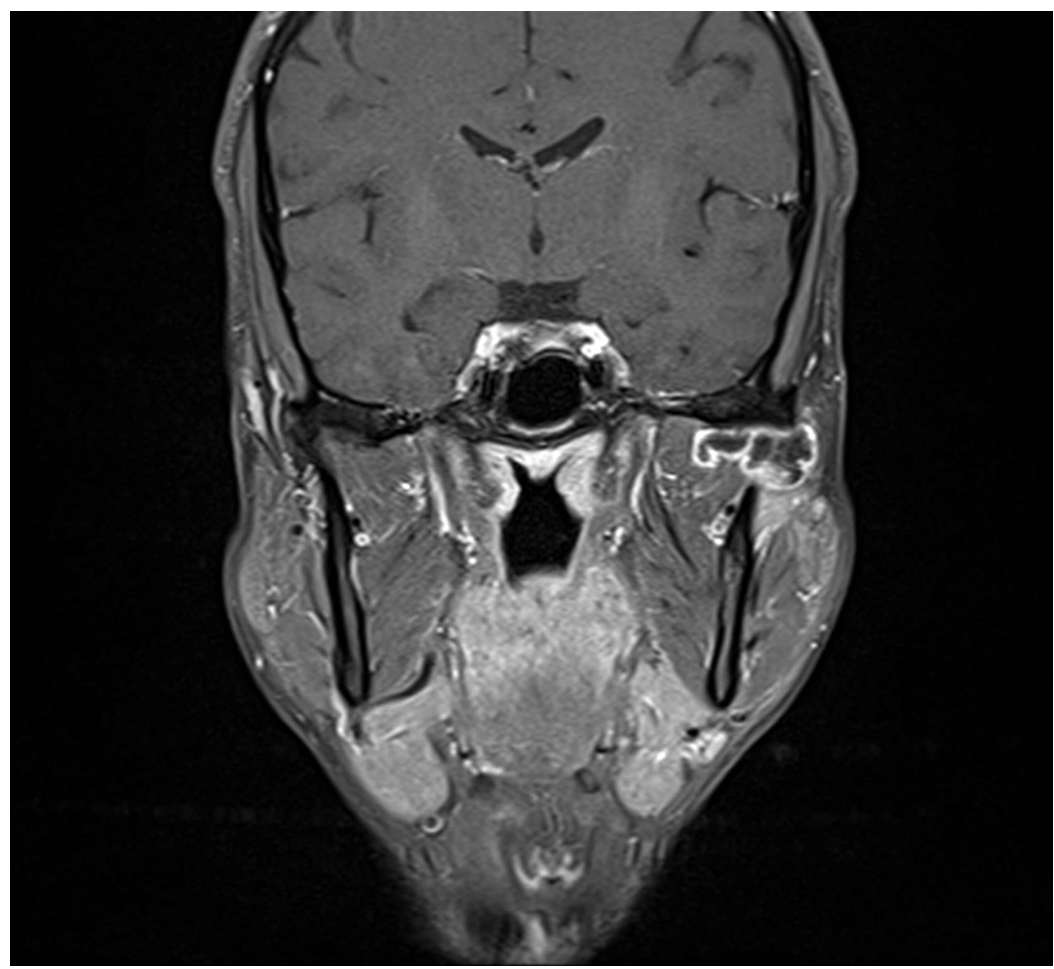

Figure 1. Heterogeneous hyposignal on T1 MRI sequences in the left temporomandibular joint. MRI: magnetic resonance imaging. 


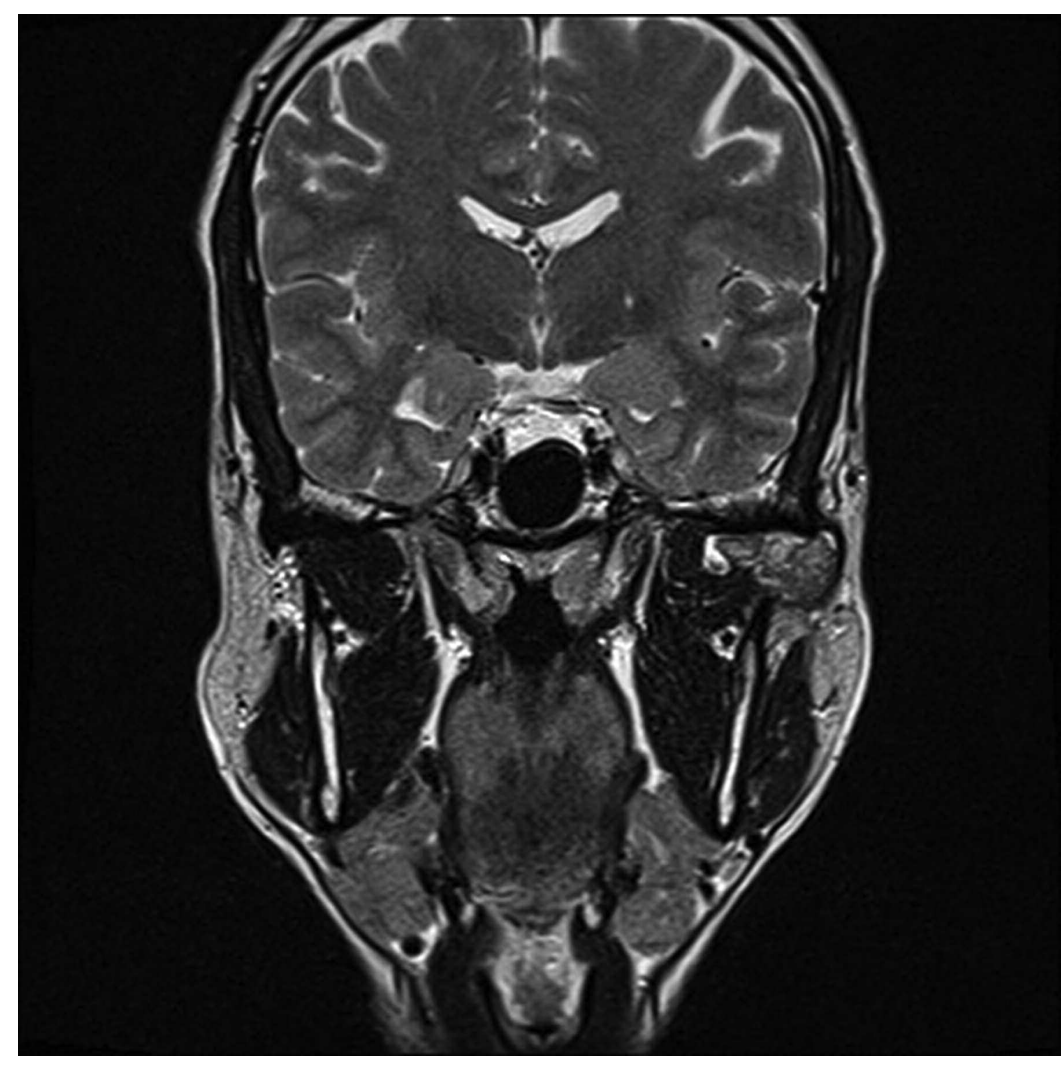

Figure 2. Heterogeneous hyposignal on T2 MRI sequences in the left temporomandibular joint. MRI: magnetic resonance imaging.

variable T2 signal) and can appear similar to other soft tissue masses $^{3}$.

Our cases demonstrate the heterogeneous presentation of gout in an unusual and single location without hyperuricemia and with heterogeneous T1 and T2 hyposignal.

\section{REFERENCES}

1. Bhattacharyya I, Chehal H, Gremillion H, Nair M. Gout of the temporomandibular joint: a review of the literature. J Am Dent Assoc 2010;141:979-85.
2. Zhang W, Doherty M, Pascual E, Bardin T, Barskova V, Conaghan $\mathrm{P}$, et al; EULAR Standing Committee for International Clinical Studies Including Therapeutics. EULAR evidence based recommendations for gout. Part I: Diagnosis. Report of a task force of the Standing Committee for international Clinical Studies Including Therapeutics (ESCISIT). Ann Rheum Dis 2006; 65:1301-11.

3. Forbess LJ, Fields TR. The broad spectrum of urate crystal deposition: unusual presentations of gouty tophi. Semin Arthritis Rheum 2012;42:146-54 